\title{
Aspectos físicos do solo em diferentes sistemas de manejo como indicador de sustentabilidade
}

\author{
Bruna Stephane Nascimento da Silva ${ }^{a}$, Ellen Patrícia Nascimento ${ }^{a}$, Victória Oliveira \\ Assunção Limaa , Larissa Carvalho de Oliveiraa; Nássila Ribeiro Galinaa; Gleidson \\ Marques Pereirab.
}

aGraduando do Curso de Engenharia Ambiental, Universidade do Estado do Pará (UEPA), Campus de Marabá, PA, Brasil.
cProfessor na Universidade do Estado do Pará (UEPA), Campus de Marabá, PA, Brasil.

RESUMO Com objetivo de avaliar as características físicas dos solos em áreas sob diferentes usos no Projeto de Desenvolvimento Sustentável Porto Seguro, localizado no município de Marabá - PA, foram utilizados indicadores físicos do solo, como a densidade do solo que influencia diretamente na densidade de partículas e porosidade do solo. Foram coletadas amostras de solo na camada de 0 a $15 \mathrm{~cm}$ pelo método de anel volumétrico (MAV), segundo Embrapa (1997) de áreas pertencentes a três diferentes usos (mata nativa, sistema agroflorestal - SAF e cultivo de mandioca). Os resultados foram submetidos à análise de variância ANOVA e a comparação entre as médias foram feitas pelo teste de Tukey a 5\% com auxílio do programa PAST. O SAF implementado não só ajudou na proteção como melhorou as qualidades físicas do solo, aumentando o fornecimento de matéria orgânica que ajuda na formação de agregados e, por conseguinte, ajuda a conservar os macroporos que são importantes para o processo de infiltração.

PALAVRAS-CHAVE: assentamentos rurais; física do solo; qualidade do solo.

Aceito 14 de novembro de 2020 Publicado online 29 de dezembro de 2020

Cite este artigo: Silva et al. (2021) Aspectos físicos do solo em diferentes sistemas de manejo como indicador de sustentabilidade. Multidisciplinary Science Journal 3: e2021001, doi: 10.29327/multiscience.2021001.

\section{Physical aspects of the soil in different management systems as an indicator of sustainability}

ABSTRACT In order to assess the physical characteristics of soils in areas under different uses in the Porto Seguro Sustainable Development Project, located in the municipality of Marabá - PA, physical soil indicators were used, such as soil density that directly influences particle density and soil porosity. Soil samples were collected in the 0 to $15 \mathrm{~cm}$ layer by the volumetric ring method from areas belonging to three different uses (native forest, agroforestry-SAF system and cassava cultivation) using the volumetric ring method (MAV), according to Embrapa (1997). The results were submitted to ANOVA analysis of variance and the comparison between the averages was made by the Tukey test at $5 \%$ with the aid of the PAST program. The implemented SAF not only helped in the protection but also improved the physical qualities of the soil, increasing the supply of organic matter that helps in the formation of aggregates and, therefore, helps to preserve the macropores that are important for the infiltration process.

KEYWORDS: rural settlements; soil physics; soil quality.

\section{Introdução}

Segundo Vezzani e Mielniczuk (2009) a qualidade do solo pode ser conceituada como a capacidade do mesmo funcionar em quaisquer tipos de ecossistema, mantendo a produtividade, a qualidade dos meios naturais (água, ar, terra e etc.) e a saúde dos seres vivos que rodeiam esse solo. Os pioneiros desse tipo de estudo foram Doran e Parkin 
(1994), que dividem os indicadores em: biológicos, físicos e químicos, sendo eles a textura, profundidade, densidade, temperatura, $\mathrm{pH}$, teores de $\mathrm{C}$ e $\mathrm{N}$ orgânico total, teores de $\mathrm{N}$ mineral, entre outros. Alterações nesses atributos podem afetar a qualidade do solo, uma vez que influencia na atividade biológica, disponibilidade de nutrientes e decomposição da matéria orgânica do solo (Dexter 2004).

Entre as propriedades físicas mais avaliadas está a densidade, que fornece indicações a respeito do estado de compactação do solo (Silva et al 2000). A densidade do solo é, conforme Klein (2008) uma relação entre massa de solo seco e seu volume, sendo, portanto, afetada por modificações na estrutura e no arranjo e volume dos poros. O objetivo desse estudo foi coletar a densidade do solo em uma visita técnica no Projeto de Desenvolvimento Sustentável (PDS) Porto Seguro, contribuindo para uma análise mais abrangente acerca do fenômeno em questão à qualidade física do solo e sua relação com os impactos gerados pelo seu manejo.

\section{Material e Métodos}

O PDS Porto Seguro está localizado na BR - 155, zona rural do município de Marabá, sudeste do Pará (Figura 1), com longitude de 49 02' 041" W e latitude de 5²8' 134" S de Greenwinch, altitude média de 85m, com clima tropical, classificado de clima Aw segundo a Köppen e Geiger. Possuindo o solo Argissolo de textura argilosa de acordo com os critérios do Sistema Brasileiro de Classificação de Solos (Embrapa 2006).

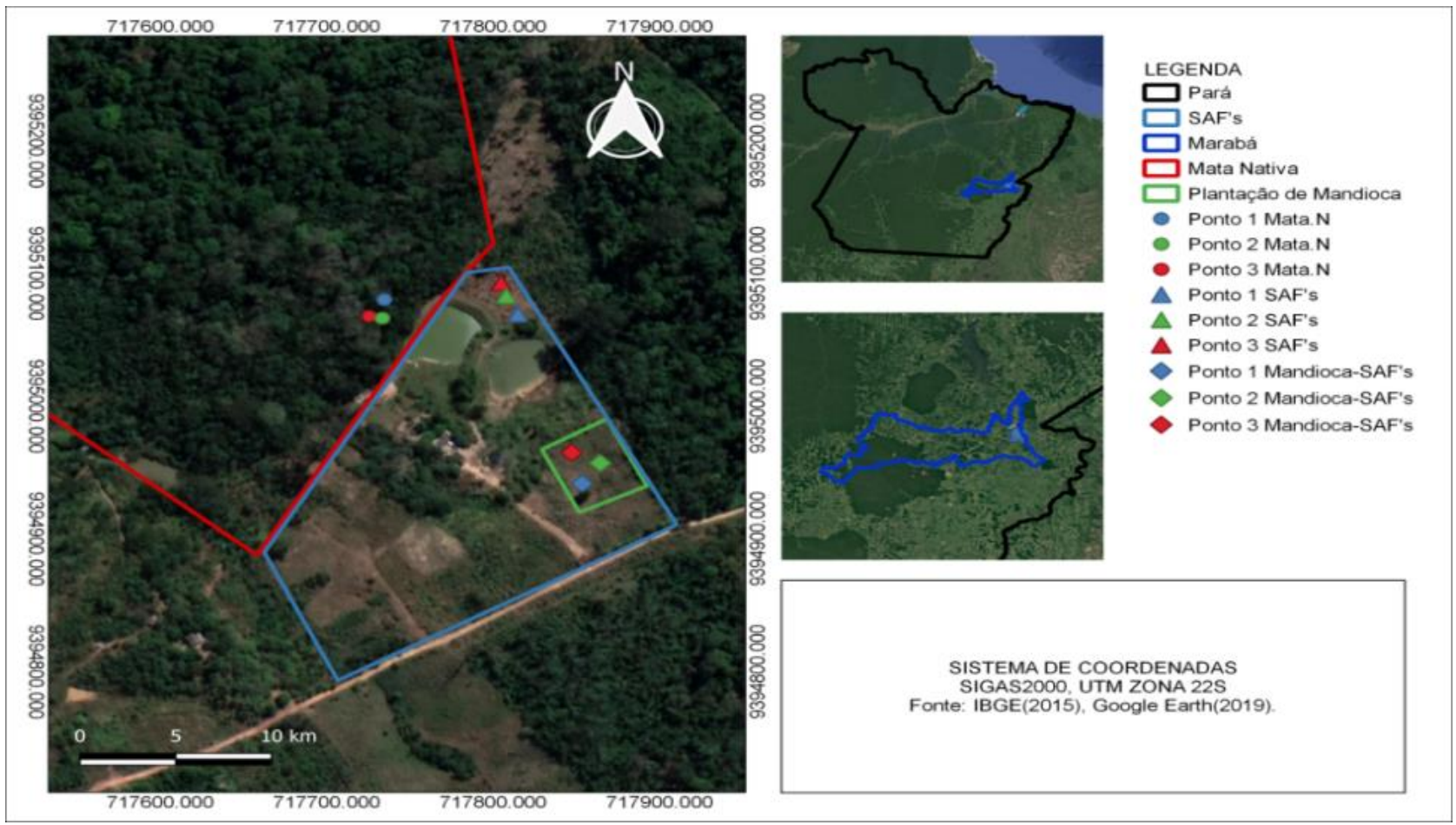

Figura 1 Mapa de localização dos pontos coletados no PDS Porto Seguro.

Para realização de análise de densidade do solo (DS) em laboratório, foram coletadas amostras do solo na propriedade do PDS em um ponto central de cada parcela (tratamentos: Cultivo de Mandioca - CM, Mata Nativa - MN e Sistema Agroflorestal - SAF) com limpeza da superfície do terreno, utilizando o método de anel volumétrico (MAV), segundo Embrapa (1997).

A coleta ocorreu em abril de 2019, em uma visita técnica de campo, nas camadas de 0-5 cm, 5-10 cm e $10-15 \mathrm{~cm}$ (Figura 2). Em cada parcela foram coletadas 9 amostras para determinação da DS em cada profundidade. Foram coletados torrões com aproximadamente $66 \mathrm{~cm}^{3}$ para determinação do anel volumétrico, conforme Embrapa (1997). As amostras foram retiradas em cilindros de aço com bordas biseladas (diâmetro de 5,0 cm, altura de 5,0 cm e volume de $98,125 \mathrm{~cm}^{3}$ ).

Os resultados foram submetidos à análise de variância ANOVA e a comparação entre as médias foram feitas pelo teste de Tukey a 5\%, com a utilização do programa PAST (Hammer 2017). 


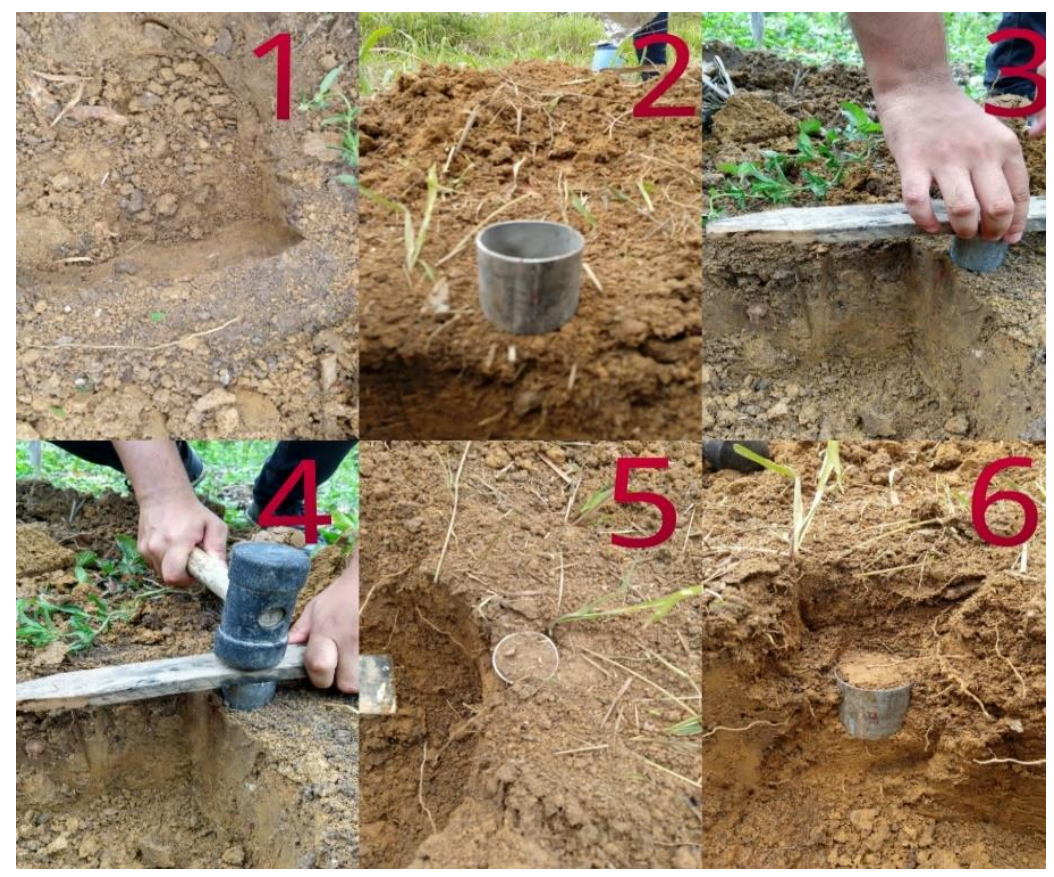

Figura 2 Procedimentos com os anéis volumétricos, 1 - abertura da cova de $0-20 \mathrm{~cm}$; 2- limpeza superficial na lateral e posicionamento do 1 o cilindro; 3- posicionamento de estaca de madeira sobre o cilindro; 4- com auxílio de uma marreta de borracha pressiona-se o cilindro sobre o solo; 5 - coleta dos primeiros $5 \mathrm{~cm}$ de solo; 6 - coleta das demais profundidades de solo.

\section{Resultados e Discussão}

Nas figuras 3, 4 e 5 estão expostos a variância de densidade do solo DS $(\mathrm{g} / \mathrm{cm} 3)$, em relação aos resultados obtidos nas três áreas distintas de estudo.

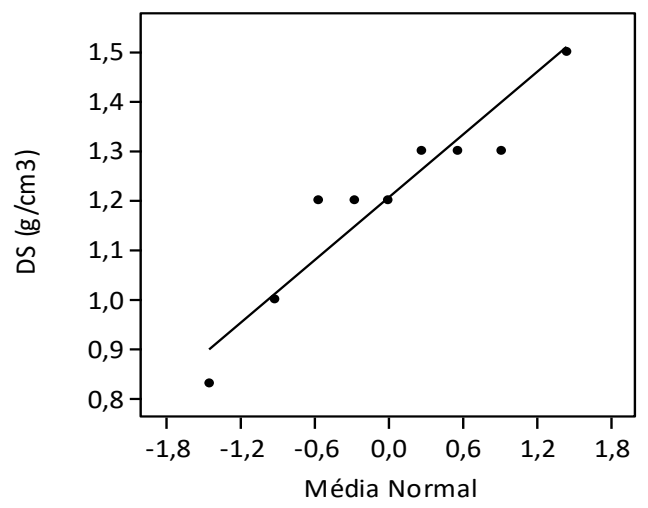

Figura 3 Variância de densidade do solo DS (g/cm3). Mata Nativa.

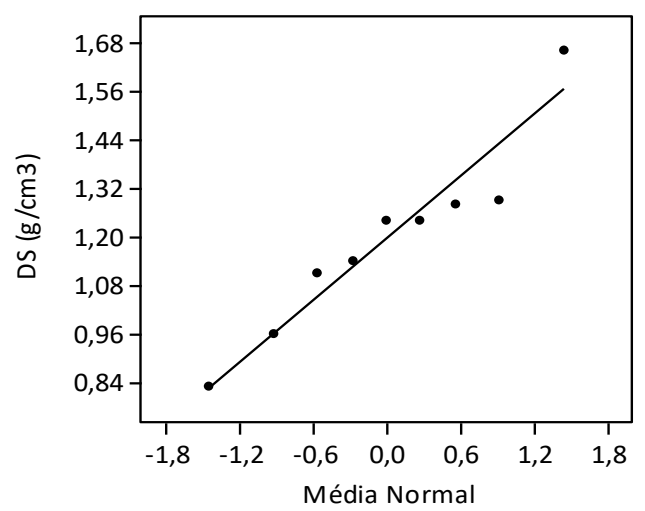

Figura 4 Variância de densidade do solo DS (g/cm3). Cultivo de Mandioca. 


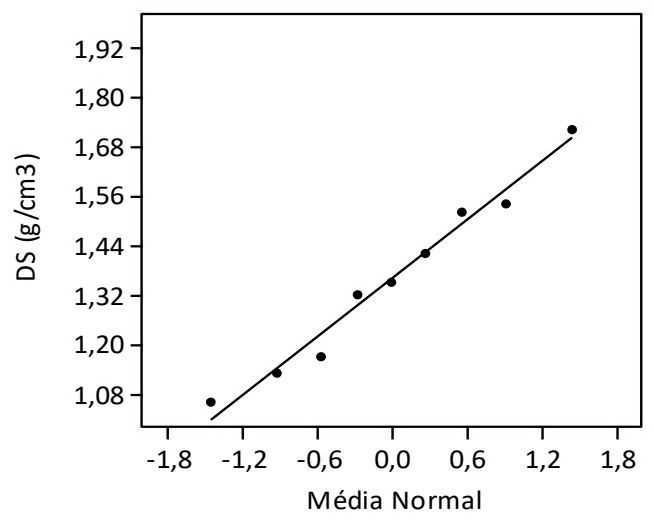

Figura 5 Variância de densidade do solo DS (g/cm3). SAF's.

Observa-se que a DS demonstra uniformidade nas 3 áreas de tratamento e a variância estabelecida manteve-se valores considerados próximo significativamente sem apresentar discrepância elevada em consideração a todas as vinte e sete variáveis. $O$ que significa dizer que ao comparar os três tratamentos não há uma variação significativa desta característica física, ou seja, não existem grandes diferença nas densidades do solo apresentadas entre os tratamentos em questão. $\mathrm{O}$ solo da área de pesquisa é considerado predominantemente argiloso, o que, segundo dados encontrados na literatura (Kiehl 1979) pode ter a DS média variando de 1 à $1,25 \mathrm{~g} / \mathrm{cm}^{3}$. Desta forma, quanto mais argiloso é o solo, menor será a sua densidade.

Com bases nos resultados obtidos, pode-se aferir que a área de estudo MN se enquadra dentro dos padrões esperados para este tipo de solo, mostrando apenas na última camada, de $11-15 \mathrm{~cm}$, uma DS um pouco superior à média literária. Isso é justificado devido ao aumento de profundidade e características do solo local, pois o mesmo é classificado quanto ao tipo de solo, de acordo com a classificação regional, com ausência de pesquisa precisa na área analisada. Desta forma, esta informação serve de referência para as demais áreas de tratamento no local. Por outro lado, o SAF apresenta a DS superior ao considerado normal pela literatura, pois de acordo com Fonseca et al (2018), a DS normal para SAF deve estar entre os valores de 0,7 a $1,2 \mathrm{~g}_{\mathrm{cm}} \mathrm{cm}^{-3}$. Se ultrapassar esses valores o sistema pode ser associado à compactação, com alta probabilidade de oferecer riscos de restrição ao crescimento radicular. No entanto, levando em consideração a DS considerada ideal para solos argilosos, os valores não discreparam tanto.

Nota-se que na área reservada para o cultivo de mandioca, todas as médias das profundidades analisadas se mantiveram dentro dos padrões ideais (Kiehl 1979). Segundo Vieira et al (2007), é comum o cultivo de espécies como a mandioca, na região amazônica, para fins comerciais e de subsistência. Para Fonseca et al (2018), a DS na área de cultivo de mandioca está dento dos valores permitidos de 0,7 a 1,2 g. $\mathrm{cm}^{-3}$, para a região amazônica.

Na tabela 1, encontra-se a densidade do solo (DS) registrada pelo teste de médias de Tukey $(P<0,05)$ em todas as áreas da pesquisa.

Tabela 1 Densidade do solo em diferentes camadas e diferentes tratamentos.

\begin{tabular}{cccc}
\hline Tratamentos & Camada de 0-5 $(\mathrm{cm})$ & Camada de 5-10 $(\mathrm{cm})$ & Camada de 10-15 $(\mathrm{cm})$ \\
\hline MN & $1,11 \mathrm{aA}$ & $1,16 \mathrm{aA}$ & $1,33 \mathrm{aA}$ \\
$\mathrm{SAF}$ & $1,33 \mathrm{aA}$ & $1,4 \mathrm{aA}$ & $1,38 \mathrm{aA}$ \\
$\mathrm{CM}$ & $1,16 \mathrm{aA}$ & $1,14 \mathrm{aA}$ & $1,25 \mathrm{aA}$ \\
\hline
\end{tabular}

Legenda: MN - Mata nativa; SAF - Sistema agroflorestal; CM - Cultivo de mandioca. Médias seguidas de letras minúsculas e maiúscula iguais não diferem estatisticamente. Médias seguidas de letras maiúscula e minúsculas diferentes são significativas estatisticamente pelo teste de Tukey a 5\% de probabilidade.

Com bases nos resultados expostos na Tabela 1, não houve interação significativa entre os tratamentos para nenhuma das profundidades estudadas. Não foram verificadas diferenças entre os locais MN, CM e SAF. Contudo, as melhores médias estão nos primeiros $5 \mathrm{~cm}$ de solo. Estes dados são corroborados por Luciano et al (2010) e Castro et al (2012), os quais verificaram aumento da densidade do solo com o aumento na profundidade em avaliações de áreas de floresta nativa, enquanto em áreas com sistemas silvo pastoris, este mesmo comportamento foi verificado por Dalben e Osaki (2008) e Martini et al (2014). 
De acordo com a EMBRAPA (2006), um valor alto de densidade do solo indica alta resistência à penetração do solo, sendo considerados "solos pesados" ou adensados e podem ser solucionados com manejo adequado, com incremento de matéria orgânica e conservação da água. A DS influencia diretamente a porosidade total do solo pela movimentação de água e ar através dos macroporos, bem como na retenção de água pelos microporos (Teixeira et al 2009).

O SAF obteve médias mais elevadas em todas as profundidades. Isto pode ser devido às características edafológica que apresentam muitos agregados inconsolidados. No CM, verificou-se que uma boa média tem relação com a forma de manejo da cobertura morta da pós-colheita utilizado para incorporação de biomassa. Ou seja, o sistema de plantio direto que minimiza as perdas do solo, reduz os custos e melhora a qualidade do solo.

\section{Conclusões}

Não foi verificado efeito de degradação oriundo do manejo com os tratamentos estudados quanto a características física do solo analisada. Os valores médios de DS demonstraram serem maiores na área do Sistema Agroflorestal, no entanto isso não significa degradação e sim característica do solo em questão. Assim, o SAF implementado não só ajudou na proteção como melhorou as qualidades físicas do solo, aumentando o fornecimento de matéria orgânica que ajuda na formação de agregados e, por conseguinte, ajuda a conservar os macroporos que são importantes para o processo de infiltração. Concomitante a isso, ajuda na diversidade de culturas, aproveitando melhor a energia e possibilita uma melhoria nos atributos físicos do solo. $\mathrm{O}$ tipo de manejo pode diminuir o uso de fertilizantes e ajuda na manutenção da qualidade do solo da região. Possivelmente, nos próximos anos, o SAF apresentará cada vez mais, melhores índices de DS, se aproximando ainda mais da MN.

\section{Referências}

Castro MA, Cunha FF, Lima SF, Paiva VB, Leite DAP, Magalhães FF (2012) Atributos físico-hídricos do solo ocupado com pastagem degradada e floresta nativa no Cerrado Sul-MatoGrossense. Brazilian Geographical Journal: Geosciences and Humanities Research Medium 3: 498-512.

Dalben AD, Osaki F (2008) Atributos físicos do solo de um cambissolo hápliom floresta nativa e de Pinus taeda. Revista Acadêmica de Ciências Agrárias e Ambientais 6:29-37.

Dexter AR (2004) Soilphysicalquality. Part I. Theory, effectsofsoiltexture, densityandorganicmatter, andeffectson root growth. Geoderma 120:201-214.

Doran JW, Parkin TB (1994) Defining and assessing soil quality. In: Doran JW, Coleman DC, Bezdicek DF, Stewart BA (eds) Defining soil quality for a sustainable environment. Madison, SSSA, pp 1-20.

Embrapa (2006) Sistema Brasileiro de Classificação de Solos. Brasília, 306p. Disponível em: https://www.agrolink.com.br/downloads/sistema-brasileiro-de-classificacao-dos-solos2006.pdf. Acesso em: 4 de junho de 2019.

Embrapa (1997) Manual de métodos de análise de solo. 2.ed. Brasília, 212p. Disponível em: https://www.agencia.cnptia.embrapa.br/Repositorio/Manual+de+Metodos_000fzvhotqk02wx5ok0q43a0ram31wtr.pdf. Acesso em: 04 de junho de 2019.

Fonseca RR, Barbosa KSS, Jesus KE, Silva LM, Sousa NTB, Silva GR, Pauletto D, Otake MYF (2018) Densidade e umidade do solo em sistemas agroflorestais. Agroecossistemas 10:367-3741.

HAMMER, $\varnothing$ (2017) Paleontologica Lst atistics Version 3.15. Reference manual. Natural History Museum, University of Oslo, 253 p. Disponível em: http://eeb.lu.lv/ftp/pub/TIS/datu_analiize/PAST/3.xx/past-3.15-manual.pdf. Acesso em: 23 de maio de 2019.

Kiehl EJ (1979) Manual de edafologia: Relação solo-planta. Piracicaba, Ceres, 262p. Disponível em: https://biblioteca.incaper.es.gov.br/busca?b=ad\&id=4455\&biblioteca=vazio\&busca=autoria:\%22KIEHL,\%20E.\%20J.\%22\&qFacets=a utoria:\%22KIEHL,\%20E.\%20J.\%22\&sort=\&paginacao=t\&paginaAtual=1. Acesso em: 1 de dezembro de 2020.

Klein VA (2008) Física do solo. Passo Fundo: EDIUPF, 212p. Disponível em: http://editora.upf.br/index.php/component/content/article/26-livros/76-fisica-do-solo-3. Acesso em: 01 de dezembro de 2020.

Luciano RV, Bertol I, Barbosa FT, Kurtz C, Fayd JÁ (2010) Propriedades físicas e carbono orgânico do solo sob plantio direto comparado a mata natural, num Cambissolo Háplico. Revista de Ciências Agrovetetinárias 2010:9-19.

Martini IC, Rosa DP, Trindade FS, Silva KPX, Lunedo P, Marcilli G (2014) Propriedades físicas de um Nitossolo sob diferentes usos de exploração agropecuária. RAMVI Getúlio Vargas 2014:1-12. 
Silva VR, Reinert DJ, Reichert JM (2000) Comparação entre métodos do cilindro e do torrão na determinação da porosidade e da densidade do solo. Ciência Rural, Santa Maria 30.

Teixeira W, Fairchild TR, Toledo MD, Taioli F (2009) Decifrando a Terra. [S.1: s.n.]. Disponível em: https://bdpi.usp.br/item/001806435. Acesso em: 04/06/2019.

Vezzani FM, Mielniczuk J (2009) Uma visão sobre qualidade do solo. Revista Brasileira Companhia do Solo 33.

Vieira TA, Rosa LS, Vasconcelos PCS, dos Santos MM, Modesto RS (2007) Sistemas agroflorestais em áreas de agricultores familiares em Igarapé-Açu, Pará: caracterização floristica, implantação e manejo. Acta Amazônica 37:549-558. 